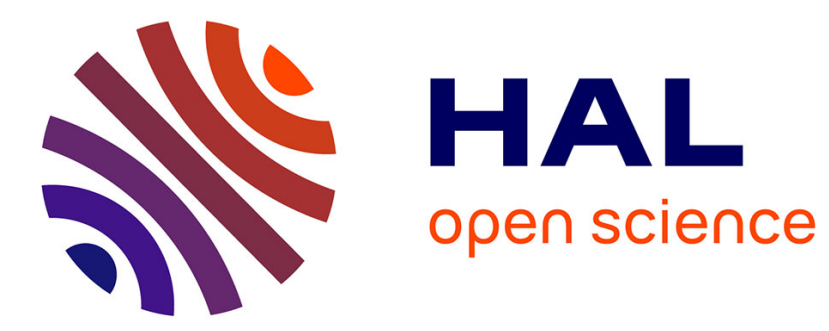

\title{
L'algèbre en Inde au XIIe siècle
}

François Patte

\section{To cite this version:}

François Patte. L'algèbre en Inde au XIIe siècle. Sciences et médecine en Asie en hommage à Jean Filliozat, Nov 2006, Paris, France. pp.83. hal-00382531

\section{HAL Id: hal-00382531 \\ https://hal.science/hal-00382531}

Submitted on 8 May 2009

HAL is a multi-disciplinary open access archive for the deposit and dissemination of scientific research documents, whether they are published or not. The documents may come from teaching and research institutions in France or abroad, or from public or private research centers.
L'archive ouverte pluridisciplinaire HAL, est destinée au dépôt et à la diffusion de documents scientifiques de niveau recherche, publiés ou non, émanant des établissements d'enseignement et de recherche français ou étrangers, des laboratoires publics ou privés. 


\section{Académie des Inscriptions et Belles Lettres en hommage à Jean Filliozat 17 novembre 2006}

L'algèbre en Inde au XII ${ }^{\mathrm{e}}$ siècle

François Patte - EFEO 
Dans l'Inde classique, Jean Filliozat écrit : "L'algèbre est attestée chronologiquement après l'œuvre de Diophante (IV ${ }^{e} s$.). Il se pourrait donc qu'elle en dérivât. Cependant il n'existe pas de preuve positive de cette dérivation et l'algèbre indienne ne ressemble pas par ses procédés à celle de Diophante qui d'ailleurs n'employait pas de notation algébrique mais seulement des abréviations dans les phrases de ses raisonnements... ${ }^{\prime \prime}$

Parmi les procédés utilisés par l'algèbre des Grecs, il y a l'usage de raisonnements fondés sur la géométrie et une référence constante à cette discipline - que l'on songe à la dénomination de nombres carrés ou cubes pour désigner le produit d'une quantité par elle-même - dont les mathématiciens auront du mal à s'affranchir, interdiction étant faite de mêler, dans un calcul, des grandeurs de dimensions différentes. Descartes n'écritil pas dans ses Regulae ad directionem ingenii : "Il faut remarquer encore que par nombre de relations il faut entendre les proportions (...) que dans l'algèbre ordinaire, on cherche à exprimer par plusieurs dimensions et plusieurs figures, et dont on nomme la première racine, la seconde carré, la troisième cube, la quatrième bicarré, etc. Ces termes m'ont moi-même longtemps trompé, je l'avoue, (...) mais après beaucoup d'expériences je m'aperçus que, par cette manière de concevoir les choses, je n'avais rien découvert que je n'eusse pu connaître bien plus facilement et distinctement sans elle; et qu'il faut rejeter entièrement de telles dénominations, de peur qu'elles ne troublent la pensée (...). Il faut donc noter avant tout que la racine, le carré, le cube, etc. ne sont que des grandeurs en proportion continue... ${ }^{2}$ "

Rien de cela dans la mathématique développée par les mathématiciens indiens qui calculent et mettent au point des algorithmes extrêmement sophistiqués pour résoudre les problèmes, sans faire appel à la géométrie, même si cette branche des mathématiques ne leur est pas inconnue. Nous allons illustrer ce fait par quelques exemples, mais auparavant, il nous faut faire deux remarques sur le titre de cette communication : "L'algèbre en Inde au $\mathrm{XII}^{e}$ siècle. »

D'abord, à propos du mot nommant cette branche des mathématiques - algèbre -, on peut remarquer que les mathématiciens européens ont emprunté ce nom à l'arabe : al-jabr, du titre d'un ouvrage du mathématicien al-Khwārizmī : al-jabr wa'l muqābala, ces mots désignant des procédures pour résoudre des problèmes. Les Indiens appellent cette discipline bijagaṇita; nous en donnerons l'explication plus loin.

Pourquoi le XII ${ }^{\mathrm{e}}$ siècle? Parmi les nombreux textes sanskrits traitant des mathématiques, nous est parvenu en entier l'ouvrage d'un mathématicien et astronome - les deux disciplines étant étroitement liées en Inde — dont

1. L'Inde classique, $\$ 1709$; EFEO, 1996

2. Descartes, Règles pour la direction de l'esprit, règle XVI ; traduction de Georges Le Roy, Bibliothèque de la Pléiade 
la vie couvre presque entièrement ce siècle : Bhāskarācārya, dit Bhāskara II, 1114, ca 1185.

Bhāskara occupe dans l'histoire des mathématiques en Inde une position centrale pour deux raisons :

1. Son œuvre condense l'ensemble des connaissances accumulées dans ce domaine au cours des siècles précédents.

2. Son œuvre est au centre des traités de mathématiques qui seront écrits, que ce soit sous forme de commentaires ou sous forme d'œuvres originales, au cours des siècles suivants et cela jusqu'au $\mathrm{Xx}^{\mathrm{e}}$ siècle.

Ce livre, le Siddhāntaśiromaṇi, le «diadème sur le Siddhānta», est un traité de mathématique et d'astronomie divisé en quatre parties :

- Lìlāvatì: Arithmétique (pāțìganita).

- Büjaganita:Algèbre.

- Grahaganita:Calcul [du mouvement] des planètes.

- Golādhyāya:Traité sur les sphères.

La partie qui nous intéresse ici, le Bïjagaṇita, fait suite à une autre qui est un traité de calcul élémentaire où sont enseignées les règles de base du calcul, la numération et les opérations, et aussi tout ce qui peut servir dans les transactions de la vie courante : calculs d'intérêts, de surfaces, de volumes, etc. Ces deux divisions sont consacrées exclusivement aux mathématiques.

Les deux parties suivantes traitent de l'astronomie et appliquent les méthodes enseignées précédemment aux calculs qui intéressent cette discipline; on voit réapparaître les mathématiques dans le Golädhyāya, où Bhāskara explique la trigonométrie et donne les formules d'addition pour les sinus et cosinus; il semble qu'il ait été le premier à le faire, du moins sous une forme quasiment exhaustive.

\section{Le Bījaganita}

\section{Le nom}

Bïja-ganita est un mot composé : bijja, la graine, l'origine, et gaṇita, le calcul, ce qui est compté; on pourrait donc traduire bijagaṇita par «calcul qui est la graine [des mathématiques] » ou « calcul sur les causes premières». Les commentateurs indiens donnent davantage de précisions; ils disent que ce calcul particulier contient en lui l'origine de tous les autres calculs, ceux faits sur les nombres, le pātìganita (arithmétique), comme la graine, cette petite unité, contient en elle toute la diversité de l'être vivant à l'origine duquel elle se trouve. Certains font même la comparaison avec l'état indifférencié du monde avant sa création, avyakta, qui contient en puissance toute 
la diversité du monde créé, manifesté, vyakta; les deux types de calculs portant aussi les noms d'avyaktagaṇita (algèbre) et de vyaktagaṇita (arithmétique) ${ }^{3}$.

\section{La discipline}

Voici ce que dit Bhāskara à la fin de son Siddhāntaśiromaṇi : asti trairāśikam pātī bījạ̣ ca vimalā matị̣| kim ajñātam subuddhīnām ato mandārtham ucyate \|

Le pāṭì (arithmétique) est proportion et le bīja (algèbre), pure pensée; qu'y a-t-il d'inconnu pour qui est intelligent? Ceci est donc dit pour les lents [d'esprit].

Dans cette strophe, Bhāskara nous indique la différence entre le pāțigaṇita et le bijaganita; l'absence de commentaire est cruelle, mais on peut penser que là où ces deux disciplines se croisent, quand il s'agit de déduire, à partir de données, une quantité a priori inconnue — pour tout dire, résoudre une équation, sujet principal de l'algèbre -, le bijaganita, grâce à la notion d'inconnue, nous entraîne vers l'abstraction, la pure pensée, tandis que le pạțiganita - des exemples de la Lìlāvatī nous le montrent - nous maintient dans un calcul pratique sur des nombres, en remplaçant l'inconnue par une valeur arbitraire, qui permet de trouver la solution par une simple règle de proportion. ${ }^{4}$

Mais :

naiva varṇātmakaṃ bījam na bījāni pṛthak pṛthak | ekam eva matir bījam analpā kalpanā yatạ̣ \|

Le bīja n'est pas [réduit] à la nature des couleurs (inconnues), il n'y a pas plusieurs bīja séparés les uns des autres, le bīja est une unité, pensée, parce que la création mentale n'est pas de petite envergure.

Il ne faut donc pas croire que le bijjaganita peut se réduire à la simple notion d'inconnue, aux $x$ et aux $y$; le bijaganita est une unité, l'abstraction qui permet de concevoir mentalement une situation concrète; il est ce saut de la pensée qui permet de passer d'une multiplicité de calculs, chacun s'appliquant à une situation particulière, à un seul concept permettant d'obtenir la solution d'une multiplicité de problèmes mathématiques concernant différentes situations.

3. Voir les différentes interprétations du mañgala (strophe propitiatoire) que Bhāskara écrit au début du Bỉjagaṇita, données par le commentateur Sūryadāsa. Le Siddhāntaśiromani I-II ; Droz, 2004 ; pp. 195-202.

4. Ainsi avons-nous traduit le mot trairāśika de la strophe qui désigne, stricto sensu, la règle de trois, parce que les exemples donnés font plus largement appel à des notions de proportion. 
Quel est le contenu de cette discipline? Nous ne donnerons pas ici une table des matières exhaustive du traité de Bhāskara, mais quelques exemples des problèmes abordés, en présentant des « exercices » proposés à l'étudiant qui se prépare à devenir mathématicien comme au mathématicien chevronné.

Nous avons essentiellement deux types d'énoncés : ceux posant une question mathématique, comme :

Quel est le nombre dont le produit du carré par huit, augmenté de un, est lui-même un carré?

Sous cette forme très simple qui présente un problème très facile à résoudre de prime abord, se cache une grosse difficulté : la solution demandée doit être un nombre entier; ce problème a, en fait, une infinité de solutions : comment, en connaissant une, trouver les autres? Le chapitre qui traite cette question nous donne la manière de les calculer toutes.

Ou bien, on trouvera une question concrète qu'il faudra traduire mathématiquement - modéliser, dirait-on aujourd'hui - avant de la résoudre :

Quelqu'un possède trois cents unités d'une monnaie et six chevaux; un autre dix chevaux du même prix mais une dette de cent unités; les deux sont également riches. Quel est le prix d'un cheval?

Ce qui est proposé dans cet exemple, c'est la résolution d'équations à une inconnue.

À nouveau, un problème formulé sous forme mathématique :

Quel nombre multiplié par douze et ajouté à son cube est égal à six fois son carré augmenté de trente-cinq?

Nous avons ici une équation du troisième degré à une seule inconnue. C'est un problème difficile qui demande une technique élaborée. Notons que ce type d'équation a été abordé, entre autres, par le poète et astronome persan Omar Khayyām, l'auteur des Rubayāt, au XI ${ }^{\mathrm{e}}$ siècle, qui aboutit à une impasse en tentant de résoudre ces équations simplement par le calcul et proposa des solutions s'appuyant sur des modèles géométriques. Les méthodes de résolution de ces équations se développeront en Europe au XVI siècle, avec Jérôme Cardan dans son traité l'Ars Magna, entièrement consacré à l'algèbre.

Autre exemple :

Les chevaux de quatre personnes sont respectivement cinq, trois, six et huit; les chameaux, deux, sept, quatre et un; les mules, huit, deux, une et trois et les moutons, sept, un, deux et un. Elles sont également riches. Dis le prix des chevaux etc.

Ce problème met en jeu la résolution simultanée de plusieurs équations à plusieurs inconnues, ici quatre; l'absence du montant de la richesse de chacun, produit une infinité de solutions. 


\section{L'usage}

Nous avons vu que le Bïjaganita était une section d'un ouvrage consacré aux mathématiques et à l'astronomie; l'usage naturel de l'algèbre est donc l'astronomie et l'utilisation principale de cette dernière, à cette époque, est l'astrologie. Nombre de mathématiciens-astronomes sont souvent des astrologues de cour.

Il est d'une très grande importance pour l'astrologue de savoir calculer avec précision, en observant le mouvement des corps célestes, des séries de jours, fastes ou néfastes - pour des cérémonies : intronisations, mariages —, de prévoir des éclipses etc.

Dans ses articles sur l'astronomie indienne, Jean Filliozat insiste à plusieurs reprises sur le fait que les Indiens ont construit un modèle très précis pour le repérage des mouvements astronomiques : les nakșatra, série de vingt-sept ou vingt-huit constellations qui permettent de repérer la position de la lune et, par voie de conséquence, celle du soleil qui, lors de la pleine lune, se trouve en opposition diamétrale. Jean Filliozat fait remarquer que, d'une part, disposer de vingt-sept repères - par rapport aux douze du Zodiaque - augmente la précision des mesures, mais que, d'autre part, le fait de pouvoir faire les observations la nuit donne une plus grande exactitude puisque la lumière du soleil éteint les constellations qui servent à noter sa position.

On peut aussi constater ce souci de précision des Indiens en algèbre pour la description des procédures de calcul et leur mise en œuvre concrète sur une planche couverte de poussière ou sur le sable sur lequel on trace les étapes du calcul.

Les règles construites font le plus souvent appel à des procédés itératifs qui donnent à leur expression une très grande concision ${ }^{5}$. Le procédé de l'itération peut paraître banal aujourd'hui, mais, à y bien réfléchir, il suppose une faculté d'analyse très minutieuse de la procédure qui l'emploiera. De même que les lettrés indiens se sont penchés avec acuité sur la langue et l'écriture pour décomposer celles-ci en petites unités minimales signifiantes, de même les mathématiciens - mais ce sont sans doute les mêmes - ont poussé l'analyse des processus des calculs pour les décomposer en petites unités que l'on pourra utiliser de manière répétée jusqu'à épuisement d'une étape avant de passer au processus suivant.

Nous allons voir, mais sans entrer dans les détails mathématiques ou astronomiques qui sous-tendent la procédure, comment Bhāskara décrit la résolution d'un certain type d'équations en nombres entiers.

5. Nous ne parlons pas ici de la formulation dans la langue, le sanskrit, qui dans tous ces traités écrits en vers, montre une recherche pour aboutir à une très grande économie de phrases et de mots. 
Dans un commentaire à l'œuvre de Bhāskara datant $\mathrm{du} \mathrm{Xv}$ e siècle, le commentateur, voulant illustrer par un exemple l'usage astronomique de ce type d'équations et leur résolution, nous dit : "Au vendredi 15 de la quinzaine claire du mois de Bhädrapada, en l'année 1342 de l'ère śaka, au cours de mon travail sur le vénérable Siddhāntaśiromaṇi, (...) la position du soleil en signes du zodiaque, etc., est de 1972948521 révolutions du soleil, 4 signes du zodiaque, 26 degrés, 27 minutes, 52 secondes et le reste de secondes est de $1374375600000 »$.

À partir de ce reste de secondes, notre commentateur veut déterminer le nombre de jours écoulés depuis le début du présent kalpa, depuis la création du monde. Pour cela, il faut trouver les solutions en nombres entiers d'une série d'équations dont la première est celle-ci, exprimée en utilisant nos notations :

$$
60 r-1374375600000=1577916450000 s
$$

Mais il ajoute aussitôt : "En raison de la lourdeur de ce calcul, l'auteur ${ }^{6}$ expose un exemple avec des nombres plus petits. » Et il propose de résoudre l'équation suivante ${ }^{7}$ :

$$
60 x+6=11 y
$$

Nous allons présenter les règles de Bhāskara et la disposition employée dans les manuscrits indiens pour résoudre cette équation. Nous mettrons à droite nos notations actuelles, à gauche les notations employées en Inde et au centre la progression des résultats obtenus en suivant les prescriptions de Bhāskara. Nous essaierons aussi de mettre en valeur le caractère itératif des règles en citant celles-ci petit à petit et, éventuellement, en en répétant une partie.

On peut remarquer que cette équation a une solution "évidente" : le couple $x=1, y=6$, mais, comme nous l'avons dit précédemment, ce type d'équation a une infinité de solutions et la méthode employée est une méthode générale pour trouver un couple de solutions dont on peut déduire tous les autres.

La disposition initiale est la suivante :

$$
\begin{aligned}
& \text { bhā } 60 \text { kṣe } 6 \\
& \text { hā } 11
\end{aligned} \mid \quad 60 x+6=11 y
$$

Bhāskara appelle dividende le nombre 60 et diviseur le nombre 11; ce sont les abréviations des noms en sanskrit de ces termes que l'on voit dans

6. C'est-à-dire le commentateur lui-même, les auteurs indiens se paraphrasant volontiers eux-mêmes.

7. Qui, à part le nombre 60 - il y a des constantes auxquelles on ne peut échapper, les degrés, minutes, secondes mesurant les angles, appartenant à un système sexagésimal — n'a rien à voir avec l'équation précédente. 
le formalisme utilisé par les Indiens : bha = bhäjya (dividende) et $h \bar{a}=h \bar{a} r a$ (diviseur) ; quant à kṣe, c'est l'abréviation du mot kṣepa qui signifie «additif».

Règle : On divisera mutuellement ces dividende et diviseur irréductibles, jusqu'à ce qu'il y ait l'unité à la place du dividende.

On voit apparaître le côté itératif de la règle par l'indication : «jusqu'à ce que... »; le reste va se mettre à la place du dividende — c'est ainsi que l'on procède quand on écrit sur le sable : le résultat vient remplacer la donnée sur laquelle a porté le calcul - et nous placerons ce quotient au milieu en anticipant sur la suite de la règle :

Règle: On divisera mutuellement ces dividende et diviseur irréductibles, jusqu'à ce qu'il y ait l'unité à la place du dividende. Les quotients devront être placés l'un au-dessous de l'autre.

Ceci nous donne :

$$
\begin{array}{r}
5 \\
11
\end{array} \mid \begin{array}{r}
5 \\
\end{array}
$$

La condition «jusqu'à ce qu'il y ait l'unité à la place du dividende» n'étant pas remplie - il y a cinq — nous recommençons l'opération; le terme $d i$ vision mutuelle est un terme technique qui indique qu'une fois la première étape achevée, c'est le diviseur précédent que l'on divise par le reste obtenu. On obtient donc la disposition suivante :

$$
\begin{array}{r}
11 \\
5
\end{array} \mid 5 \quad 60=5 \times 11+5
$$

À nouveau :

Règle: On divisera mutuellement ces dividende et diviseur irréductibles, jusqu'à ce qu'il y ait l'unité à la place du dividende. Les quotients devront être placés l'un au-dessous de l'autre.

$$
\begin{aligned}
& 1 \\
& 5
\end{aligned} \mid \begin{array}{ll}
5 & 60=5 \times 11+5 \\
\end{array}
$$

Nous en avons maintenant fini avec cet algorithme puisque le reste 1 a pris la place du dividende. Nous pouvons poursuivre l'application de la règle :

Règle: On divisera mutuellement ces dividende et diviseur irréductibles, jusqu'à ce qu'il y ait l'unité à la place du dividende. Les quotients devront être placés l'un au-dessous de l'autre, au-dessous de cela doit être placé l'additif puis, à la place du dernier, zéro.

Nous pouvons donc effacer les opérations qui ont servi à constituer le début de la disposition nécessaire au calcul des solutions de l'équation et placer 6 au-dessous du 2 : 
Puis :

Tout est en place maintenant pour appliquer l'algorithme qui va nous conduire à la solution du problème, mais, auparavant, il convient de faire une remarque au sujet de ce zéro qui vient se placer à la base de cette colonne, de cette liane (vallī) comme l'appellent les mathématiciens indiens.

D'abord le signe « 0 » est employé en Inde pour marquer un emplacement vide dans une suite de caractères que l'on écrit et, si cela est sans doute son origine en tant que «zéro " mathématique - il marque le chiffre manquant à une place dans l'écriture d'un nombre en notation décimale - , zéro est toujours considéré comme à part parmi les nombres. Un commentateur du Bïjagnita, Sūryadāsa, écrit même au XVI ${ }^{\mathrm{e}}$ siècle que zéro n'est pas du domaine des nombres par lui-même $e^{8}$ et les opérations concernant zéro ne sont pas traitées en même temps que celles sur les nombres, mais font l'objet d'un chapitre séparé dans la Lìlāvatī comme dans le Bïjagnita. Il n'en reste pas moins qu'ici, zéro va naturellement faire partie des calculs qui vont suivre.

Ensuite, quand on analyse du point de vue mathématique - ce que nous ne ferons pas ici - comment fonctionne l'algorithme qui est utilisé, on ne peut que penser que l'idée de mettre zéro à cette place est un trait de génie, car les calculs qui vont maintenant nous conduire aux solutions s'en trouvent simplifiés à l'extrême. Cette méthode, qui porte en sanskrit le nom de kuttaka (pulvérisateur), est connue depuis le $\mathrm{v}^{\mathrm{e}}$ siècle au moins; jusqu'à ce texte de Bhāskara, la méthode voulait qu'à partir d'un certain moment, dans la constitution de la "liane», on mît un nombre arbitraire pour procéder aux calculs qui vont suivre. Le Bījagṇita de Bhāskara est le premier texte à utiliser zéro à cette place dans cette méthode.

Venons-en maintenant à la règle suivante qui va nous permettre de calculer les solutions de l'équation. Comme la règle précédente, celle-ci est itérative et nous emploierons les mêmes conventions de notations, laissant toutefois de côté l'expression actuelle des calculs qui sont extrêmement simples.

Règle : L'avant-dernier ayant multiplié celui qui est au-dessus de lui

8. Le Siddhāntaśiromṇi, I-II; Droz, 2004 ; p. 214. 
$6 \longleftarrow$ avant-dernier

0

donc, en remplaçant 2 par le résultat de la multiplication, on obtient :

Règle : L'avant-dernier ayant multiplié celui qui est au-dessus de lui [puis], le dernier ayant été ajouté, on supprimera le dernier; en renouvelant, on aura un couple de nombres.

On voit apparaître la prescription d'itération : «en renouvelant, on aura un couple de nombres. » Nous avons trois nombres dans notre «liane», il faut donc recommencer l'opération :

Règle : L'avant-dernier ayant multiplié celui qui est au-dessus de lui

$$
\begin{aligned}
& 5 \longleftarrow \\
& 12 \longleftarrow \text { avant-dernier } \\
& 6
\end{aligned}
$$

60 , résultat de la multiplication, vient remplacer 5, et :

Règle : L'avant-dernier ayant multiplié celui qui est au-dessus de lui [puis], le dernier ayant été ajouté, on supprimera le dernier; en renouvelant, on aura un couple de nombres.

Nous obtenons maintenant un couple de nombres : la procédure est terminée et ces deux nombres sont la solution de notre équation :

$$
60 \times 12+6=726=11 \times 66
$$

Comme le nombre des solutions de ces équations est infini, Bhāskara nous donne le moyen de les calculer : il suffit de prendre chaque élément du couple solution que cette méthode nous a permis de calculer - 66 et 12 
- et de lui ajouter un multiple du multiplicateur de l'autre dans l'équation. Ainsi, quand on prend 66, on lui ajoute un multiple du multiplicateur de 12 et réciproquement :

$66+60 \times t$ et $12+11 \times t$ sont donc encore des solutions si on prend le même nombre pour $t$ en calculant un nouveau couple.

De cette manière, en prenant $t=1$ :

$126=66+60 \times t$ et $23=12+11 \times t$ est encore un couple de solutions :

$60 \times 23+6=1386=126 \times 11$.

Ou encore, si $t=-1$ :

$6=66+60 \times(-1)$ et $1=12+11 \times(-1)$ sont les solutions « évidentes » que nous avons signalé au début. 


\section{Solutions des exercices donnés en exemple}

Premier exemple :

Quel est le nombre dont le produit du carré par huit, augmenté de un, est lui-même un carré?

Nous avons dit que ce problème avait une solution très simple et, de fait, on voit facilement que : $8 \times 1^{2}+1=9=3^{2}$.

Plus difficile est de donner toutes les solutions du problème général : $8 \times x^{2}+1=y^{2}$ - il y en a une infinité ! - et Bhāskara donne une règle pour les construire dès qu'on en connaît une; ici, le couple de nombre 1 et 3 .

Voici cette règle. Bhāskara appelle petite [racine] le nombre $x$, et grande [racine], le nombre $y$; le coefficient, ici 8 , s'appelle la prakrti, et le nombre que l'on ajoute, ici 1, l'additif.

Ayant posé la «petite», la "grande» et l'additif [puis] placé respectivement au-dessous d'eux les mêmes, ou d'autres, de multiples racines sont calculées, grâce à leur composition: soit le produit en croix des "grandes" et des "petites", leur somme est la [nouvelle] «petite»; et le produit des deux "petites", multiplié par la prakrti, ajouté au produit des "grandes", est la [nouvelle] "grande»; dans ce cas, le produit des deux additifs sera l'additif.

La description donnée propose une disposition comme celle qui suit; les manuscrits ajoutent souvent le coefficient (la prakrti). Comme nous ne disposons que d'une solution, le couple $(1,3)$, nous plaçons «les mêmes » sur la deuxième ligne :
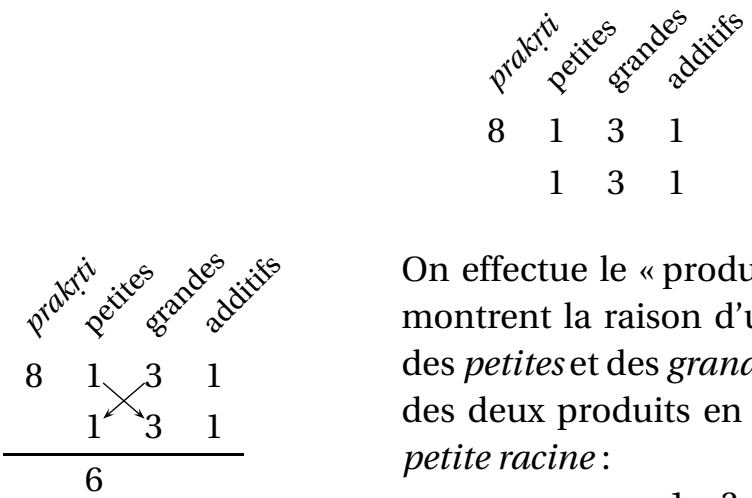

On effectue le "produit en croix » — les flèches montrent la raison d'une telle dénomination des petites et des grandes, puis on place la somme des deux produits en dessous, comme nouvelle petite racine:

$$
1 \times 3+3 \times 1=6
$$

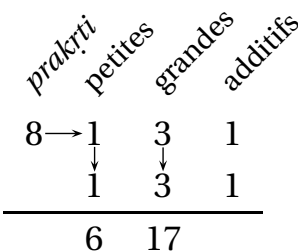

Nous effectuons le produit des petites et de la prakrti, puis le produit des grandes et nous plaçons la somme des deux comme nouvelle grande racine:

$$
1 \times 1 \times 8+3 \times 3=17
$$


La règle prescrit aussi de faire le produit des additifs, mais dans notre cas cela n'est pas nécessaire, puisque ceux-ci sont égaux à "un », le nouvel additif sera lui aussi «un».

Nous avons ainsi obtenu un nouveau couple de solutions $(6,17)$ :

$$
8 \times 6^{2}+1=289=17^{2}
$$

Pour trouver d'autres solutions, on disposera, cette fois-ci, «les autres » - le couple que l'on vient de calculer - sur la deuxième ligne et on appliquera la même règle de calcul :

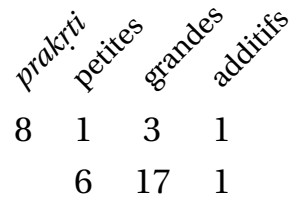

On trouve ainsi un nouveau couple, $(35,99)$ :

$$
8 \times 35^{2}+1=9801=99^{2}
$$

Et ainsi de suite... Telle est l'infinité, concluent souvent les commentaires.

Deuxième exemple :

Quelqu'un possède trois cents unités d'une monnaie et six chevaux; un autre dix chevaux du même prix mais une dette de cent unités; les deux sont également riches. Quel est le prix d'un cheval?

"Diophante n'employait pas de notation algébrique... ", nous faisait observer Jean Filliozat; les Indiens ont développé un formalisme pour noter les "inconnues" en leur donnant, à l'exception de la première, des noms de couleurs. Ainsi, ce que nous appelons, et notons, $x$, ils l'appellent yāvattāvat, "autant que», et le noteront $y \bar{a} ; y$ est appelé kālaka, "noir», noté $k \bar{a} ; z$ est appelé nīlaka, «bleu », noté $n \bar{l} ; t$ est appelé pìta, «jaune», noté $p \bar{\imath}$; pour différencier les simples nombres des coefficients qui affectent les inconnues, ils les feront précéder de " $r \bar{u} »$, abréviation de rūpa, unité. En revanche, il n'y a aucun signe pour les opérations; une addition se notera par une simple juxtaposition et une soustraction par l'addition d'un nombre affecté d'un caractère négatif : un point au-dessus.

Il y a une seule inconnue ici : le prix d'un cheval; Bhāskara la note donc en utilisant l'inconnue yāvattāvat et nous dit alors que la fortune du premier s'élève à :

$$
y \bar{a} 6 r \bar{u} 300
$$

et la fortune du second, puisqu'il a une dette de cent unités, à :

$$
\text { yā } 10 r \bar{u} 1 \dot{0} 0
$$


En prenant des notations qui nous sont plus familières, et en notant par $f$ la fortune de chacun, nous écririons, en appelant $x$ le prix du cheval :

$$
\begin{aligned}
6 x+300 & =f \\
10 x-100 & =f
\end{aligned}
$$

Comme l'énoncé nous dit que la fortune est la même, on peut écrire que ces deux équations sont égales :

$$
6 x+300=10 x-100
$$

ou

$$
4 x=400
$$

Un cheval vaut donc 100 unités de la monnaie. Nous avons présenté la solution de manière contemporaine, mais les textes sanskrits utilisent exactement la même méthode; la seule difficulté vient de l'expression de la solution en l'absence de signe pour les opérations et pour l'égalité.

Troisième exemple :

Quel nombre multiplié par douze et ajouté à son cube est égal à six fois son carré augmenté de trente-cinq?

Nous avons vu le formalisme adopté par les mathématiciens indiens pour noter les inconnues; ils ont aussi imaginé des notations pour le carré et le cube en utilisant la première syllabe des mots dont ils se servent pour nommer le carré : varga, "ensemble d'objets identiques", et le cube : ghana, «solide». Ainsi yāva note-t-il $x^{2}$ et yāgha, $x^{3}$.

Le problème posé est donc formalisé de cette manière, en notant le nombre inconnu par $y \bar{a}$ :

$$
\begin{gathered}
\text { yā } 12 \text { yāgha } 1 \\
\text { yāva } 6 \text { rū } 35
\end{gathered}
$$

Ces deux lignes représentent la même quantité. Ici aussi nous utiliserons le formalisme contemporain pour résoudre le problème, mais la méthode de la solution est intégralement donnée par Bhāskara.

Avec nos notations, ce problème s'écrit :

$$
12 x+x^{3}=6 x^{2}+35
$$

Bhāskara fait remarquer qu'en ôtant 8 et yāva $6\left(6 x^{2}\right)$ de chaque côté on obtient une forme remarquable : les deux membres de l'équation sont des cubes :

$$
\begin{aligned}
x^{3}-6 x^{2}+12 x-8 & =35-8 \\
x^{3}-3 \times 2 x^{2}+3 \times 2^{2} x-2^{3} & =27 \\
(x-2)^{3} & =3^{3}
\end{aligned}
$$


En prenant la racine cubique de chaque côté, le problème revient à résoudre :

$$
x-2=3
$$

Le nombre cherché est donc 5.

Quatrième exemple :

Les chevaux de quatre personnes sont respectivement cinq, trois, six et huit; les chameaux, deux, sept, quatre et un; les mules, huit, deux, une et trois et les moutons, sept, un, deux et un. Elles sont également riches. Dis le prix des chevaux etc.

Nous allons avoir besoin de tous les noms d'inconnues donnés plus haut pour exprimer mathématiquement ce problème : $y \bar{a}$ sera le prix d'un cheval, $k \bar{a}$ celui d'un chameau, $n \bar{\imath}$ le prix d'une mule et $p \bar{\imath}$ celui d'un mouton.

$\begin{array}{llllll}\text { Bhāskara exprime ainsi la fortune du premier } \quad: y \bar{a} 5 & k \bar{a} 2 & n \bar{\imath} 8 & p \bar{\imath} 7\end{array}$

$$
\begin{array}{lllll}
\text { du deuxième : } y \bar{a} 3 & k \bar{a} 7 & n \bar{\imath} 2 & p \bar{\imath} 1 \\
\text { du troisième : } y \bar{a} 6 & k \bar{a} 4 & n \bar{\imath} 1 & p \bar{\imath} 2 \\
\text { du quatrième : } y \bar{a} 8 & k \bar{a} 1 & n \bar{l} 3 & p \overline{1} 1
\end{array}
$$

Puis il remarque que ces fortunes sont égales et qu'il est donc facile d'exprimer le prix des chevaux en fonction de celui des autres animaux - au fond, d'éliminer une inconnue et une équation - en regroupant la première et la deuxième, la deuxième et la troisième, enfin la troisième et la quatrième.

Nous continuerons en utilisant nos notations. La méthode suit la méthode de Bhāskara et elle est toujours enseignée de nos jours.

$$
\begin{aligned}
5 x+2 y+8 z+7 t & =f \\
3 x+7 y+2 z+t & =f \\
6 x+4 y+z+2 t & =f \\
8 x+y+3 z+t & =f
\end{aligned}
$$

En regroupant la première et la deuxième :

$$
\begin{aligned}
5 x+2 y+8 z+7 t & =3 x+7 y+2 z+t \\
2 x & =5 y-6 z-6 t
\end{aligned}
$$

Puis la deuxième et la troisième :

$$
\begin{aligned}
3 x+7 y+2 z+t & =6 x+4 y+z+2 t \\
3 x & =3 y+z-t
\end{aligned}
$$


Puis la troisième et la quatrième :

$$
\begin{aligned}
6 x+4 y+z+2 t & =8 x+y+3 z+t \\
2 x & =3 y-2 z+t
\end{aligned}
$$

On obtient alors trois équations, que l'on peut rendre égales en multipliant la première et la troisième par 2 , la deuxième par 3 :

$$
\begin{aligned}
& 6 x=15 y-18 z-18 t \\
& 6 x=6 y+2 z-2 t \\
& 6 x=9 y-6 z+3 t
\end{aligned}
$$

On recommence alors la même méthode, en regroupant la première et la deuxième :

$$
\begin{aligned}
15 y-18 z-18 t & =6 y+2 z-2 t \\
9 y & =20 z+16 t
\end{aligned}
$$

et la deuxième et la troisième :

$$
\begin{aligned}
6 y+2 z-2 t & =9 y-6 z+3 t \\
3 y & =8 z-5 t
\end{aligned}
$$

Il ne reste plus que deux équations que l'on peut rendre égales en multipliant la deuxième par 3 :

$$
\begin{aligned}
& 9 y=20 z+16 t \\
& 9 y=24 z-15 t
\end{aligned}
$$

Soit, finalement :

$$
\begin{aligned}
20 z+16 t & =24 z-15 t \\
4 z & =31 t
\end{aligned}
$$

Nous sommes finalement amenés à résoudre un kuttaka dont la méthode a été exposée plus haut (page 8), mais ici, comme il n'y a pas d'additif, une solution immédiate est de donner à $z$ la valeur du coefficient de $t$ et à $t$ celle du coefficient de $z: z=31$ et $t=4$.

En fait, il y a une infinité de valeurs pour $z$ et $t: z=31 \times a$ et $t=4 \times a$ où $a$ est un nombre arbitrairement choisi. Nous nous limiterons aux premières valeurs trouvées, qui correspondent à $a=1$ et, pour calculer le prix d'un chameau, nous remplaçons $z$ et $t$ par ces valeurs dans l'une des deux équations qui expriment $y$ en fonction de $z$ et $t$ :

$9 y=24 z-15 t=24 \times 31-15 \times 4=684$

Donc : $y=684 / 9=76$ 
Puis, on reporte ces valeurs de $y, z$ et $t$ dans une équation qui exprime $x$ pour calculer le prix d'un cheval :

$6 x=9 y-6 z+3 t=9 \times 76-6 \times 31+3 \times 4=510$

Donc : $x=510 / 6=85$.

Le prix d'un cheval est donc 85 , d'un chameau 76 , d'une mule 31 et d'un mouton 4 . 